\title{
A single-mode fiber optic interferometric temperature sensor
}

\section{Georgina Beltrán-Pérez, Gilberto Camacho-Basilio}

Georgina Beltrán-Pérez, Gilberto Camacho-Basilio, "A single-mode fiber optic interferometric temperature sensor," Proc. SPIE 9663, Eighth International Topical Meeting on Education and Training in Optics and Photonics, 96632Q (6 October 2003); doi: 10.1117/12.2208441

SPIE Event: Eighth International Topical Meeting on Education and Training in Optics and Photonics, 2003, Tucson, Arizona, United States 


\title{
A single-mode fiber optic interferometric temperature sensor
}

\author{
Georgina Beltrán-Pérez and Gilberto Camacho-Basilio \\ Facultad de Ciencias Fisicomatemáticas, Benemérita Universidad Autónoma de Puebla \\ Av. San Claudio y 18 sur, Apdo. Postal 1152, C.P. 72000, Puebla, Pue.,México \\ telephone: 222-2295500,ext.2138 and 2128; fax:222-2295636; e-mail: gbeltran@fcfm.buap.mx and gcamacho@fcfm.buap.mx
}

\begin{abstract}
In this work we show an education experimental setup for a single-mode fiber interferometric temperature sensor based on an Mach-Zehnder array. The used fiber has a refractive index core of 1.456 for wavelength of $633 \mathrm{~nm}$. The sensor calibration was done by using ice and phase change was measured by counting of the fringes.

(C)2003 Optical Society of America

OCIS codes: (060.2370) Fiber optics sensors; (120.2650) Fringe analysis
\end{abstract}

\section{Introduction}

The sensitivity of an interferometric fiber optic sensor to temperature [1] is determined by the rate of change of optical path length $n L$ with temperature. The phase of the light wave which travels a distance, $L$, in an optical fiber is given by $\phi=\beta L$, where $\beta$ is the propagation constant of the light in the fiber. Changing any physical parameter of the fiber's environment causes a phase change given by

$$
\delta \phi=\beta \delta L+L \delta \beta .
$$

The first term on the right hand side of the equation is due to a change in the length of the fiber, while the second term is due to a change in the propagation constant. The length, $L$, now represents the length over which the physical change affects the fiber. The quantity which we actually wish to determine is the phase change per unit fiber length per unit of physical stimulus, $(\delta \phi) / S L$, where $\mathrm{S}$ is the stimulus. The magnitude of the stimulus can then be measured by counting the shift of the fringes for a fiber of known interaction length. As an example, consider the effect of a temperature change, $\delta T$, which affects a length, $L$, of the fiber in the sensor arm of the interferometer. There are two effects which occur: the change of length due to thermal expansion or contraction and the change of the propagation constant due to the temperature dependence of the index of refraction. Thus, Eq. 1 becomes [2]

$$
\delta \phi /(\delta T L)=(2 \pi / \lambda)[(n / L)(\delta L / \delta T)+(\delta n / \delta T)],
$$

This technique allows for the construction of the sensor which has high sensitivity and high resolution.

\section{Experimental Setup}

The Fig. 1 shows the experimental setup of the temperature sensor. It consists basically in a Mach-Zehnder configuration. The first beamsplitter is replaced by a bidirectional coupler and the light is launched into two singlemode fibers of equal lengths. These two fibers represent the two interferometer arms. One of the fibers serves as the reference arm, which is kept isolated from external perturbations, while the other fiber serves as the sensor arm. The phase shift is detected when the two beams are recombined at the receiver end of the sensor. It results in irterference fringes which can be detected and counted.

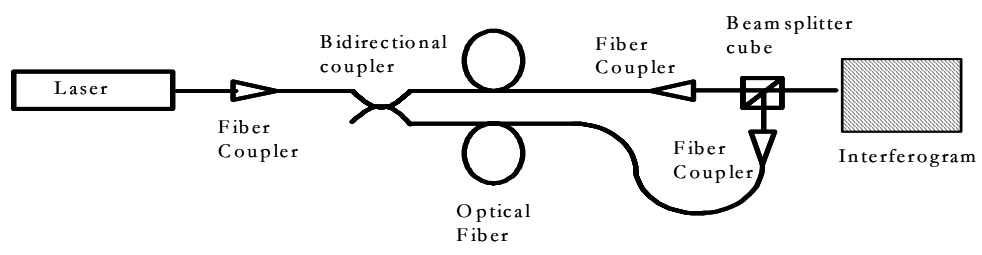

Fig. 1. Experimental setup for the temperature sensor.

\section{References}

[1] Francis T. S. Yu and Shizhuo Yin, Fiber Optic Sensors, Marcel Dekker, Inc., New York (2002)

[2] G. B. Hocker, "Fiber optic sensing of pressure and temperature", Applied Optics, 18, 1445, (1979)

Eighth International Topical Meeting on Education and Training in Optics and Photonics,

edited by Barry L. Shoop, Grover Swartzlander Jr., Proc. of SPIE Vol. 9663, 96632Q

(C) 2003 SPIE, OSA, ICO · doi: 10.1117/12.2208441 